\title{
Dysbiosis In Epizootic Shell Disease Of The American Lobster (Homarus Americanus)
}

\author{
NJ Meres \\ CC Ajuzie \\ M Sikaroodi \\ M Vemulapalli \\ Jeffrey D. Shields \\ Virginia Institute of Marine Science
}

See next page for additional authors

Follow this and additional works at: https://scholarworks.wm.edu/vimsarticles

Part of the Marine Biology Commons

\section{Recommended Citation}

Meres, NJ; Ajuzie, CC; Sikaroodi, M; Vemulapalli, M; Shields, Jeffrey D.; and Gillevet, PM, "Dysbiosis In Epizootic Shell Disease Of The American Lobster (Homarus Americanus)" (2012). VIMS Articles. 341. https://scholarworks.wm.edu/vimsarticles/341 
Authors

NJ Meres, CC Ajuzie, M Sikaroodi, M Vemulapalli, Jeffrey D. Shields, and PM Gillevet 


\title{
DYSBIOSIS IN EPIZOOTIC SHELL DISEASE OF THE AMERICAN LOBSTER (HOMARUS AMERICANUS)
}

\author{
NORMAN J. MERES, ${ }^{1 *}$ CYRIL C. AJUZIE, ${ }^{3}$ MASOUMEH SIKAROODI, ${ }^{1}$ \\ MEGHANA VEMULAPALLI, ${ }^{2}$ JEFFREY D. SHIELDS ${ }^{4}$ AND PATRICK M. GILLEVET ${ }^{\mathbf{1}}$ \\ ${ }^{1}$ Environmental Science and Policy Department, George Mason University, 4400 University Drive, \\ Fairfax, VA, 22030; ${ }^{2}$ School of Systems Biology, George Mason University, 4400 University Drive, \\ Fairfax, VA, 22030; ${ }^{3}$ Aquaculture, Freshwater and Marine Ecology Research Lab, Applied Fisheries and \\ Hydrobiology Unit, Department of Zoology, University of Jos, Nigeria; ${ }^{4}$ Virginia Institute of Marine \\ Science, PO Box 1346, Gloucester Point, VA, 23062
}

\begin{abstract}
Epizootic shell disease (ESD) in the American lobster (Homarus americanus) is continuing to affect the southern New England lobster population, and the etiology of the disease has not been well defined. We hypothesized that a dysbiotic shift in the shell microbial biofilm played a key part in the etiology of the disease. We analyzed the community structure of the surface microflora of apparently healthy and diseased lobsters using multitag pyrosequencing to correlate the abundance of key taxa within the lesions. Discriminant analysis (DA) was used to identify taxa in the microbial community that were associated with diseased and healthy states. Among the 170 bacterial taxa that were identified, 58 were helpful in determining the diseased and healthy states. The remaining 112 were not significantly different between the 2 states. The genus Aquimarina was present in high abundance in both healthy and diseased lobsters, but had a significantly higher abundance on animals in the diseased state. However, DA demonstrated that this genus does not strongly discriminate between the diseased and healthy state. Phylogenetic analysis indicated that there was significant strain diversity of this genus in all the samples analyzed. Our results indicate that the lesions seen in ESD may be viewed as being correlated with a polymicrobial component rather than being caused by a discrete pathogen.
\end{abstract}

KEY WORDS: American lobster, Homarus americanus, epizootic shell disease, bacteria, dysbiosis

\section{INTRODUCTION}

Epizootic shell disease (ESD) represents an economic problem for fishermen fishing American lobsters (Homarus americanus, Milne Edwards) in the southern New England (SNE) stock, and may indicate an ecological crisis for the lobster and other members of the benthic community in the region. The histopathology of ESD has been described by Smolowitz et al. (2005). The presence of lesions on the carapace of an infected lobster is the most visible sign of the disease. Histological examination revealed bacteria as the most common organisms in the lesions, with some protist constituents in more advanced cases. The lesions were grouped into 3 categories based on depth of the bacterial incursion, presuming that depth reflects a progressive erosion of the cuticle.

Category 1 is the least severe erosion, with shallow lesions extending into the epicuticle and exocuticle. The lateral edges of the lesions often exhibited evidence of melanization, but inflammation in the underlying connective tissue or other evidence of an immune response is rarely observed at this stage. Bacteria are found in the leading edges of the lesion, and in the crystalline chitin lattice.

Category 2 lesions are moderately deep, penetrating the calcified endocuticle. The crystalline lattice structure of the chitin take on a "pillarlike" appearance as the bacteria degrade the protein structure between the lattice crystals. The endocuticle exhibits melanization, especially in the vertical areas of bacterial incursion. Evidence of an immune response to the infection includes inflammation of the underlying cuticular

*Corresponding author. E-mail: nmeres@masonlive.gmu.edu DOI: $10.2983 / 035.031 .0206$ epithelium and "moderate numbers" of hemocytes in the tissues. Secondary invasion by small protists occurs during this stage. These organisms are apparently responsible for the degradation of the crystal lattice structure of the chitin. In some category 2 lesions, an "inflammatory cuticle" forms between the uncalcified endocuticle and the cuticular epithelium. The latter has some areas of hyperplasia and hypertrophy.

Deeper erosions into the uncalcified endocuticle are characterized as category 3 lesions. At this stage, the overlying structures of the carapace are absent, and the exposed areas are melanized. The cuticular epithelium is hyperplastic, hypertrophic, and intensely inflamed, with an increased number of hemocytes. The underlying connective tissue also exhibits signs of inflammation and immune response. The most extreme types of category 3 lesions have no more tissue than the inflammatory cuticle overlying the cuticular epithelium. In some cases, the lesions progress to ulcerations, which are characterized by a complete absence of cuticular tissue and cuticular epithelium. Degranulated hemocytes develop a pseudomembrane to cover the connective tissue. The outer layer of the pseudomembrane is necrotic and melanized.

Chistoserdov et al. (2005) isolated a novel chitinolytic bacterium in the genus Aquimarina (family Flavobacteriaceae), and identified it as a possible etiological agent in ESD lesions. Tlusty and Metzler (2012) discussed the physical appearance of prelesion formation whereas Quinn et al. (2012) identified Aquimarina 'homaria' as present in these prelesion initial stages of the disease. Given that bacteria and bacterial erosion, or necrosis of the cuticle are components of ESD, we undertook an analysis of the bacterial community of lesioned areas and compared them with unaffected areas to assess the correlation of various taxa in the microbial biofilm of diseased lobsters. 


\section{METHODS}

Cuticle samples $\left(0.5 \mathrm{~cm}^{2}\right)$ were harvested as part of the " 100 Lobsters" Project (Shields et al. 2012). The lobsters were collected from within Narragansett Bay, RI. A total of 55 lobsters had ESD whereas 47 were categorized as apparently healthy (i.e. the lobsters did not have visible ESD lesions). For this study, we did not subdivide the diseased samples into the disease categories described earlier (Smolowitz et al. 2005). The samples were shipped on dry ice and stored at $-80^{\circ} \mathrm{C}$ until processed. Three types of carapace samples were taken: those with lesions (diseased), those from lobsters with disease but were in areas free of lesions (healthy on diseased), and those from apparently healthy lobsters (healthy). The carapace samples were dissolved in EDTA and proteinase $\mathrm{K}$ to isolate all microorganisms from the surface and subsurface, and total DNA was extracted using the FastPrep Bio101 kit (Qbiogene/ MP Biomedicals LLC, Solon, $\mathrm{OH}$ ). Polymerase chain reaction (PCR) was used to amplify the bacterial genes from the first 2 hypervariable regions of the 16s ribosomal RNA using universal primers $27 \mathrm{~F}$ ( $5^{\prime}$-AGA GTT TGA TCM TGG CTC AG-3') and 355R (5'-GCT GCC TCC CGT AGG AGT-3') (InvitrogenTM Corporation, Carlsbad, CA). Length heterogeneity PCR (LHPCR) fingerprinting (Suzuki et al. 1998) was used to survey samples rapidly and standardize the community amplification. Duplicate fingerprints were performed from each DNA extraction to verify that the community amplification was reproducible. An initial survey of various regions of the lobster carapace was done using LH-PCR, and the microbial community on the cephalothorax region was relatively uniform in healthy lobsters. Because the cephalothorax is prone to the development of lesions in diseased lobsters, it was used in all subsequent analysis.

We used multitag pyrosequencing (MTPS) (Gillevet 2006) to characterize the taxa in the microbiome of the carapace samples. We generated a set of 96 fusion primers that contained emulsion PCR linkers (454 Life Sciences) and different 7-base "barcodes" on either $27 \mathrm{~F}$ or $355 \mathrm{R}$ universal $16 \mathrm{~S}$ rRNA primers. Each sample of lobster DNA was then amplified with a unique set of tagged forward and reverse $16 \mathrm{~S}$ rRNA primers, pooled, subjected to emulsion PCR, and pyrosequenced using a GSFLX pyrosequencer per the manufacturer's instructions (Roche, Branchburg, NJ). Data from each pooled sample were "deconvoluted" by sorting the sequences into bins based on the barcodes using custom PERL scripts. This technique allows the rapid sequencing of multiple samples at one time, yielding thousands of sequence reads per sample. The sequence reads were identified using the Bayesian analysis in the Ribosomal Database Project (Cole et al. 2009). We used a custom PERL script to calculate the normalized abundance of each taxa in a sample based on the total reads in that sample.

The sequence for A. 'homaria' (Chistoserdov et al. 2005) was not available for analysis. However, we aligned all the sequences that were identified as Aquimarina spp. by the RDP10 analysis, with reference sequences available in GenBank, and constructed a neighbor joining tree for the genus to observe the clustering of the various species and to determine whether a correlation exists between species of the genus and the apparent health status of the lobster.

We used the software program Quantitative Insights into Microbial Ecology (QIIME) (Caporaso et al. 2010) to compare the microbial community sequences derived by MTPS on the

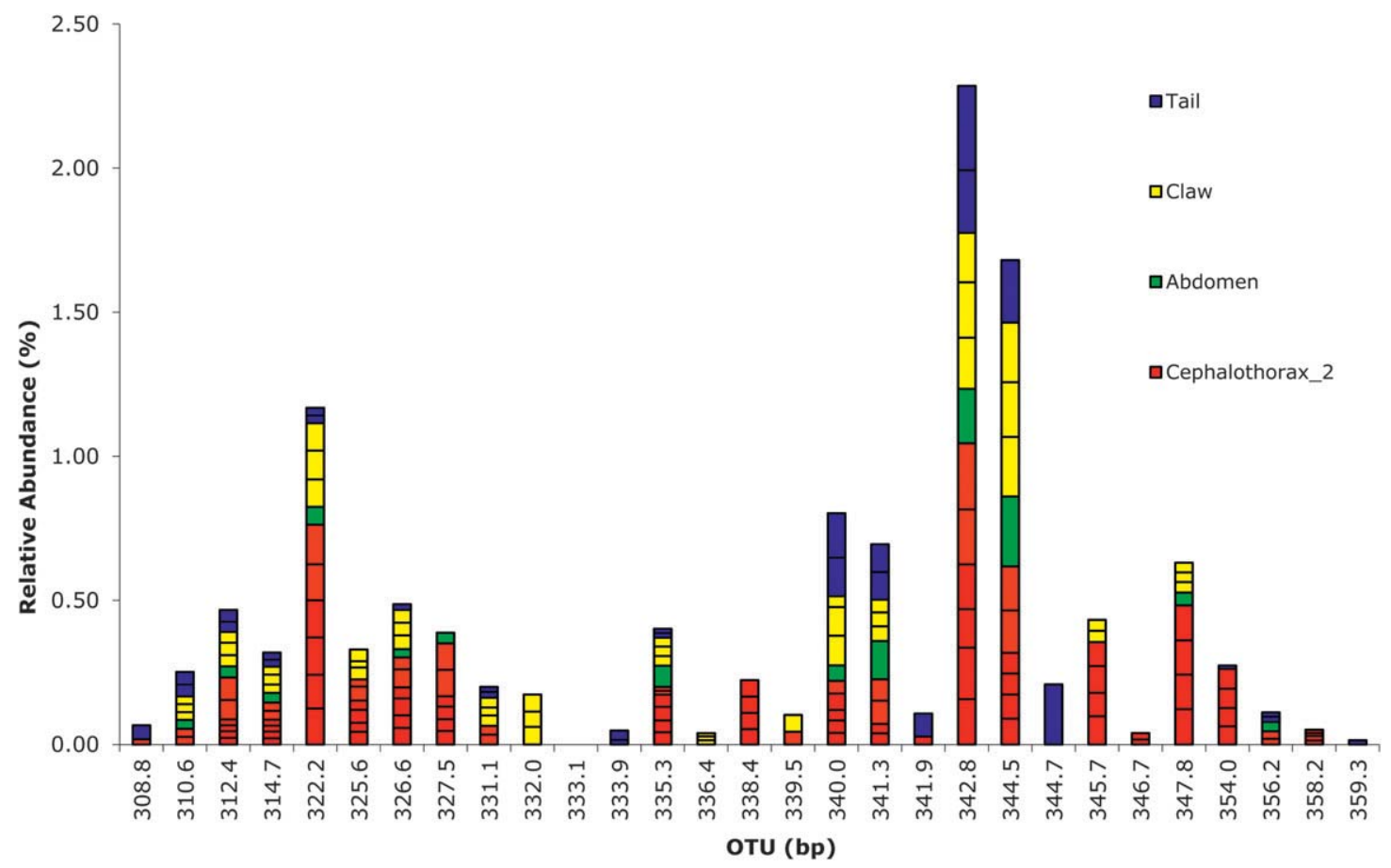

Figure 1. Replicate LH-PCR fingerprint analysis of various regions of the carapace. The peaks in the fingerprints are described as operational taxonomic units (OTUs) because we do not know what they represent (e.g., genera, species, strains). In fact, a peak may contain more than 1 species, and strains of the same species may show up in different peaks. The normalized OTU abundance for each sample was plotted as a stacked histogram to demonstrate the distribution of the microbiome on the lobster carapace. Multiple bars of the same color represent OTU abundances from several samples of the same region of the shell. Therefore, OTU abundances from multiple samples may add up to greater than $100 \%$. 
3 types of carapace samples, and constructed a Unifrac neighbor joining tree (Lozupone \& Knight 2005) that displayed graphically the similarities in the microbiome structure between each lobster sample in the study. We used PASW v.18 (IBM, Chicago, IL) for the discriminant analysis (DA) using the default parameters. Principle coordinate analysis was performed using the MultiVariate Statistical Package (Kovach Computing Services, Anglesey, Wales) using a Bray-Curtis distance metric.

\section{RESULTS}

We initially surveyed the lobster carapace to determine the bacterial community distribution of the cephalothorax, claws, abdomen, and tail. Figure 1 is a histogram of normalized abundance of operational taxonomic units (OTUs) that were present on the various regions of the carapace. The LH-PCR profiles revealed that, with a few exceptions, the OTUs present on the cephalothorax are representative of the community diversity present on other regions, such as the claw and the abdomen. However, the community diversity at the other sites was less than that on the cephalothorax, which may reflect the fact that the lobster cannot clean the carapace on the upper cephalothorax.

We then focused the investigation on the cephalothorax, because this is where the majority of the lesions occur in the disease. MTPS was performed on 102 lobster samples, and the resulting sequence reads were sorted based on their tags or barcodes. The analysis yielded 212,019 reads with an average of 1,594 reads per sample. Rarefaction analysis indicated that all samples were close to saturation. We identified 170 bacteria present on the cuticle of lobsters from Narragansett Bay, RI, having culled taxa that were of insufficient abundance to be statistically significant $(<1 \%)$. Of these 170 bacteria, 167 were identified to the level of genus, 1 was identified to the level of family, and 2 were identified as OTUs with complete identities that were unknown. Figure 2 is a histogram of average abundance of the taxa from each disease state sample rank ordered by the normalized abundance of data from the diseased lobsters. The entire histogram for all 170 taxa is depicted in the insert to Figure 2. The most abundant genera found in the carapace microbiome include Jannaschia, Aquimarina, Cardiobacterium, Thalassobius, Micrococcineae, and Loktanella. However, essentially all of these were found in all disease classes.

Figure 3 is the Principal Coordinate Analysis (PCO) of the normalized abundance of the genera identified in each disease class using a Bray-Curtis distance metric. One can see clustering of the diseased samples (red dots), which is somewhat distinct from the healthy (yellow) and healthy-on-disease samples (blue). It should be noted that the disease clusters overlap substantially, and some of the samples from one disease class are mixed in with the samples from a different disease class. For

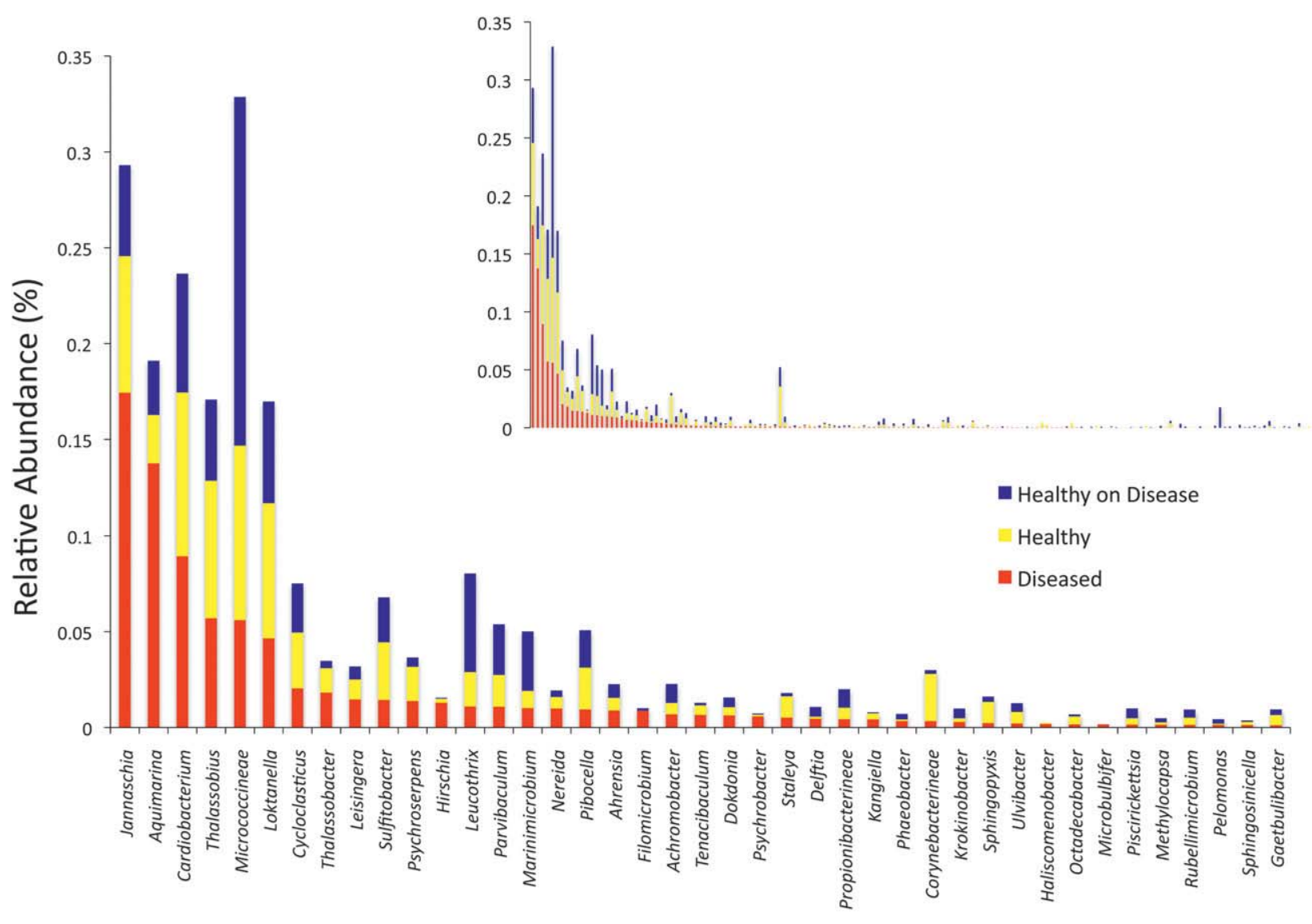

Genus

Figure 2. MTPS analysis of the cephalothorax microbiome. The normalized abundances of the most prominent taxa found on the cephalothorax of 102 lobsters is plotted as a histogram, rank ordered by those taxa in the disease state. The histogram of all 170 taxa is plotted in the insert. 


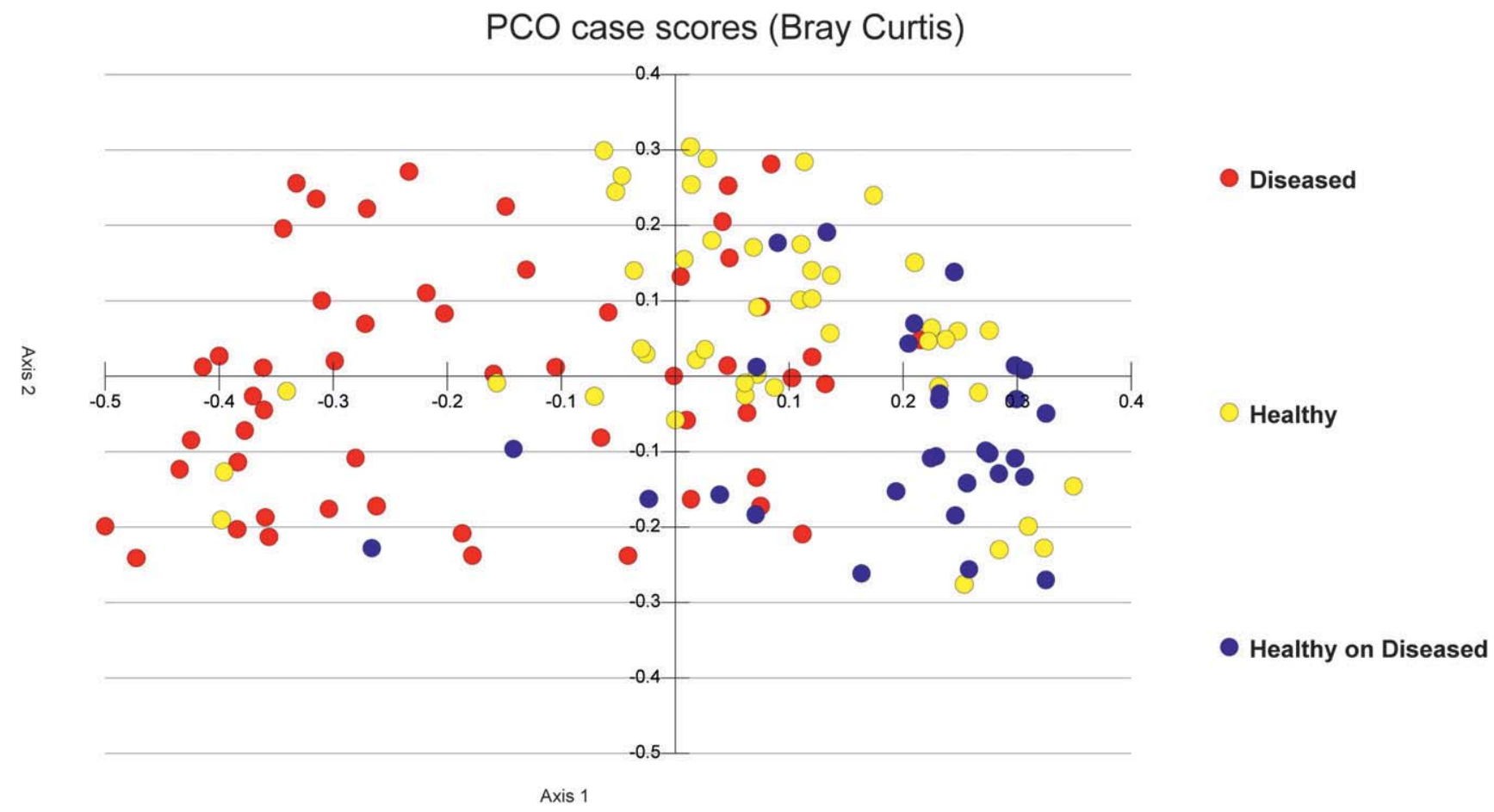

Figure 3. Principal coordinate analysis (PCO) of the normalized abundances of the genera from each disease classes. The healthy samples are yellow dots $(n=47)$, the diseased samples are red dots $(n=55)$, and the healthy-on-diseased samples are blue dots $(n=33)$.

example, a few samples from healthy lobsters are clearly within the cluster of disease samples, and there is an area of overlap among the 3 categories in the center of the chart.

A weighted Unifrac tree, built by comparing phylogenetic trees constructed using taxa richness and their corresponding abundance for each sample (Lozupone \& Knight 2005), is displayed in Figure 4. The clustering of the weighted Unifrac tree indicates the similarity and abundance of the taxa in each of the samples. This figure reveals that although there is some clustering, there is no clear separation among the 3 disease classes. That is, they are distributed more or less equally throughout the neighbor joining tree. In addition, QIIME revealed that abundance levels of alphaproteobacteria, gammaproteobacteria, and Bacteroidetes levels were elevated when comparing apparently healthy lobsters with diseased lobsters, whereas Flavobacteria (the class that includes Aquimarina spp.) were less abundant in the diseased state.

The abundance data for the 102 lobster samples and 170 variables (taxa) were analyzed by DA. Three analyses were conducted for this study: the first compared cuticle from diseased versus apparently healthy animals, the second compared cuticle from diseased animals versus healthy cuticle on diseased animals, and the third compared cuticle from apparently healthy animals versus healthy cuticle on diseased animals.

The DA for the bacterial flora on cuticle from diseased versus apparently healthy lobsters used a tolerance test to eliminated 112 variables because they lacked variance between groups and, thus, did not contribute significantly to the discriminant function (i.e., the analysis between the 2 groups relied on 58 variables (bacterial genera)). The functions at the group centroids (i.e., a multivariate equivalent to the mean) were 1.463 for disease class 1 (diseased) and -1.1712 for disease class 2 (healthy). These scores demonstrate that the centroids were well separated, which indicates that the function was discriminating between the classes $\left(R_{\mathrm{c}}{ }^{2}=0.848\right.$, with Wilks' $\lambda=0.281$, chisquare $=91.287$, and $P=0.002, d f=56)$ (Garson 2008).

The classification results (confusion matrix) of the first analysis are displayed in Table 1 . Table 2 displays the structure coefficient table. The structure coefficients are full coefficients, meaning that they are pooled, within-groups correlations between the independent variable and the standardized canonical discriminant coefficients (Garson 2008). According to Garson (2008) and Klecka (1980), the structure coefficient is the authoritative coefficient for determining the importance of the independent variable to the discriminant function. As with the standardized canonical discriminant coefficient, the absolute value represents the quantity, and the sign indicates the equality of the correlation. In the case of structure coefficients, however, the largest absolute value is 1.0. The bacteria Aquimarina spp. had a structure coefficient of 0.268 , indicating that it had a weak correlation with the function, and it ranked second to the genus Jannaschia, which had a structure coefficient of 0.325 , indicating that Jannaschia correlates more strongly with the disease state. However, these correlations are relatively low, indicating that neither is correlated strongly with the disease state.

For the second comparison, the bacterial flora on the cuticle of diseased lobsters versus healthy cuticle on diseased lobsters, the functions at the group centroids were well separated, with a value of 2.060 for disease class 1 (diseased) and -3.541 for disease class 3 (healthy on diseased; $R_{\mathrm{c}}{ }^{2}=0.939$, Wilks' $\lambda=$ 0.118 , chi-square $=131.382, P<0.001, d f=47$ ). The classification results of the second analysis are displayed in Table 3. The second section of Table 2 is the structure coefficient table for this analysis. These tables have been arranged to display 


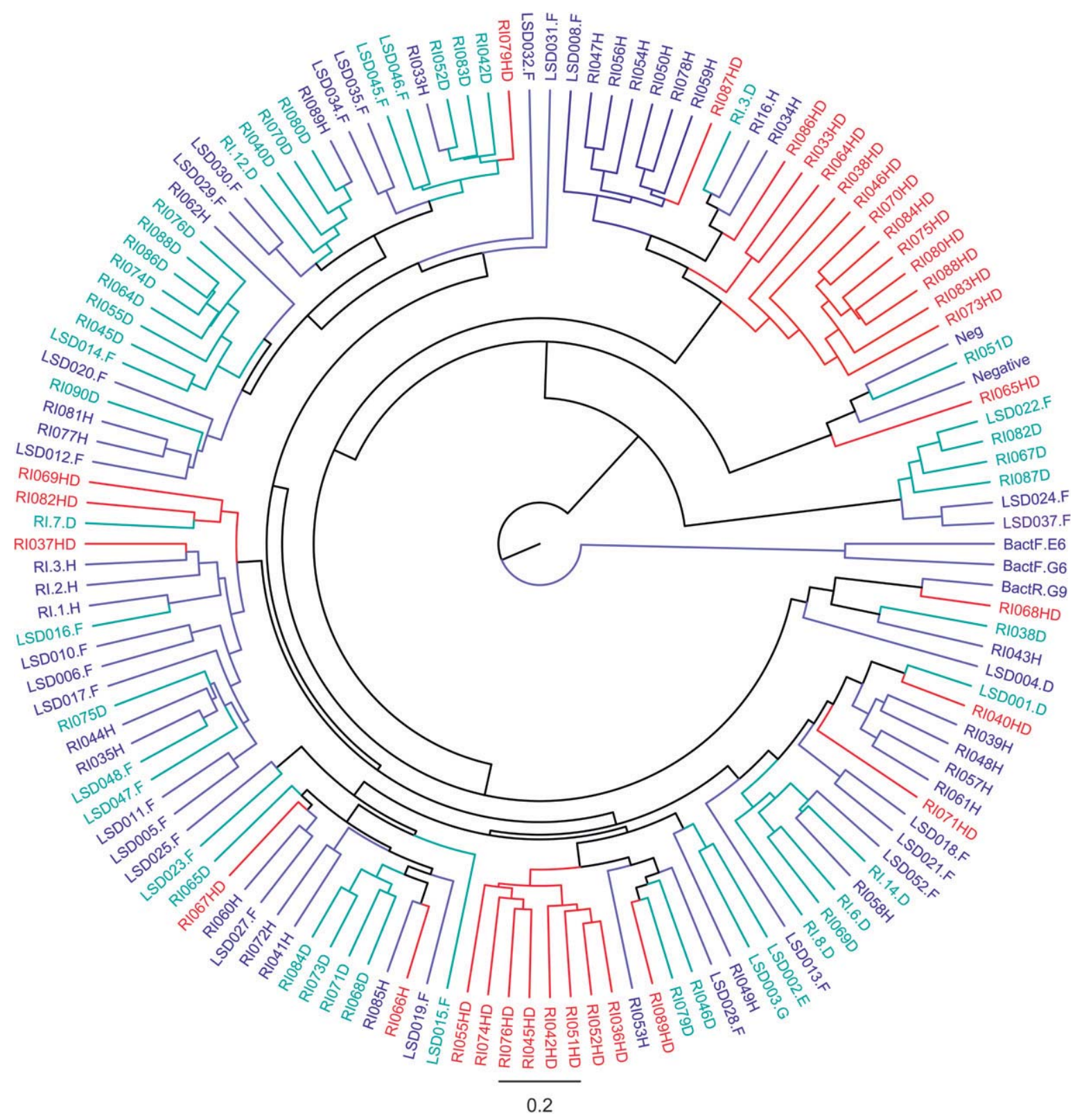

Figure 4. Weighted Unifrac tree. The disease classes are color coded. Healthy samples are blue $(n=47)$, diseased samples are green $(n=55)$, and healthy-on-diseased samples are red $(n=33)$.

descending order of importance, with the positive coefficients listed first. The negative coefficients appear to be listed in ascending order because the absolute value is indicative of a stronger correlation. Species in the genus Jannaschia had roughly the same structure coefficient as in the previous analysis. Species in the genus Aquimarina had a structure coefficient of 0.156 , and its relative ranking dropped from second to fourth in the structure coefficient table. This indicates that Aquimarina spp. appears to contribute less to the discrimination of these 2 states than they do to discriminating between the healthy and diseased states.

For the third comparison, a comparison of the bacterial flora on the cuticle of apparently healthy lobsters versus healthy cuticle on diseased lobsters, the discriminant functions at the group centroids demonstrated good separation, with values of
2.439 for disease class 2 (healthy) and -3.582 for disease class 3 (healthy on diseased; $R_{\mathrm{c}}{ }^{2}=0.948$, Wilks' $\lambda=0.100$, chi-square $=$ 122.991, $P<0.001, d f=47)$. The classification results of this analysis are displayed in Table 4 . The third section of Table 2 contains the structure coefficient table for this comparison. In this analysis, Aquimarina spp. have the lowest structure coefficient of -0.002 , indicating that it correlated weakly and negatively in discriminating between carapaces from lobsters that showed no signs of the disease and carapace samples without lesions that were taken from lobsters that have the disease.

Because the Aquimarina spp. belong to 1 of the 2 genera that had weak correlations in the previous analysis with the disease, we investigated further the species from this taxa. We aligned the sequences that were identified as Aquimarina spp. in GenBank and constructed a neighbor-joining tree (Fig. 5). A 
TABLE 1.

Classification results table (confusion matrix) for the bacterial flora on the cuticle of diseased lobsters compared with that of apparently healthy lobsters.

\begin{tabular}{|c|c|c|c|c|c|}
\hline \multicolumn{6}{|c|}{ Classification Results*: Diseased Versus Healthy } \\
\hline & & \multirow[b]{2}{*}{ Disease Class } & \multicolumn{2}{|c|}{$\begin{array}{c}\text { Predicted Group } \\
\text { Membership }\end{array}$} & \multirow[b]{2}{*}{ Total } \\
\hline & & & 1 & 2 & \\
\hline \multirow[t]{4}{*}{ Original } & Count & Diseased & 52 & 3 & 55 \\
\hline & & Healthy & 3 & 44 & 47 \\
\hline & $\%$ & Diseased & 94.5 & 5.5 & 100.0 \\
\hline & & Healthy & 6.4 & 93.6 & 100.0 \\
\hline
\end{tabular}

* A total of $94.1 \%$ of original grouped cases correctly classified. Actual group membership is compared with that predicted by the discriminant function derived from the analysis. The analysis excludes those bacteria that did not pass the tolerance test.

vast majority of the community sequences identified as Aquimarina spp. clustered as 3 clades that were separate from the reference Aquimarina spp. from GenBank. The sequences from apparently healthy, healthy on diseased (HD), and diseased lobsters (D) were present in all 3 clades. There were only a few sequences from apparently healthy and diseased lobsters that clustered with the reference sequences, indicating that the majority of the sequences represented novel species or strains.

\section{DISCUSSION}

We investigated the bacterial flora on the cuticle of lobsters with and without signs of ESD. The bacterial community was analyzed using PCO, Unifrac, and DA. For lobsters that had the disease versus apparently healthy animals from the same region, 58 genera of bacteria were sufficiently represented in the community to analyze their potential contribution in discriminating the disease state. However, according to the structure coefficient tables, no species of bacteria makes a substantial contribution to the discrimination function. The genera with the largest coefficients, Jannaschia spp. and Aquimarina spp., had weakly positive coefficients associated with the disease state (structure coefficient $=0.325$ and 0.268 , respectively). That is, ESD lesions do not appear to be correlated with any one species. Quinn et al. (2012) demonstrated that histologically similar lesions to the ones seen in ESD could be produced on lobsters when their carapace was abraded and then exposed to $A$. 'homaria.' However, this may be a feature of laboratory exposure because the field situation is much more complex with

TABLE 2.

Structure coefficient table showing the coefficients that measure the relationship with flora on the cuticle of diseased versus apparently healthy lobsters, diseased cuticle versus healthy cuticle on diseased lobsters, and healthy cuticle versus healthy cuticle on diseased lobsters.

\begin{tabular}{|c|c|c|c|c|c|}
\hline \multicolumn{2}{|c|}{ Diseased Versus Healthy } & \multicolumn{2}{|c|}{ Diseased Versus Healthy on Diseased } & \multicolumn{2}{|c|}{ Healthy Versus Healthy on Diseased } \\
\hline Taxon & $\overline{\text { Coefficient }}$ & Taxon & $\overline{\text { Coefficient }}$ & Taxon & $\overline{\text { Coefficient }}$ \\
\hline Jannaschia & 0.325 & Jannaschia & 0.315 & Jannaschia & 0.248 \\
\hline Aquimarina & 0.268 & Aquimarina & 0.156 & Leisingera & 0.127 \\
\hline Hirschia & 0.173 & Cycloclasticus & 0.125 & Corynebacterineae & 0.113 \\
\hline Oceanicolaa & 0.152 & Hirschia & 0.112 & Frankineae & 0.112 \\
\hline Methylosarcinaa & 0.148 & Corynebacterineae & 0.076 & Leucothrix & 0.094 \\
\hline Schineriaa & 0.148 & Filomicrobium & 0.073 & Cycloclasticus & 0.09 \\
\hline Shigellaa & 0.143 & Cardiobacterium & 0.066 & Hirschia & 0.063 \\
\hline Tenacibaculuma & 0.142 & Leisingera & 0.059 & Kaistia & 0.062 \\
\hline Terasakiellaa & 0.141 & Leucothrix & 0.055 & Aminomonas & 0.057 \\
\hline Filomicrobium & 0.139 & Haliscomenobacter & 0.051 & Crenothrix & 0.057 \\
\hline Thalassobactera & 0.125 & Kaistia & 0.043 & Hoeflea & 0.054 \\
\hline Microbulbifera & 0.105 & Glycomycineae & 0.043 & Erythrobacter & 0.052 \\
\hline Photobacteriuma & 0.104 & Burkholderia & 0.043 & Cardiobacterium & 0.048 \\
\hline Lacinutrix & 0.099 & Frankineae & 0.04 & Glycomycineae & 0.045 \\
\hline Streptococcusa & 0.092 & Brumimicrobium & 0.03 & Hydrogenovibrio & 0.045 \\
\hline Woodsholeaa & 0.089 & Geopsychrobacter & 0.03 & Frateuria & 0.044 \\
\hline Vibrioa & 0.073 & Agrobacterium & 0.03 & Fluviicola & 0.044 \\
\hline Haliscomenobacter & 0.065 & Fluviicola & 0.03 & Haliscomenobacter & 0.044 \\
\hline Ruegeriaa & 0.063 & Achromatium & 0.03 & Lactococcus & 0.036 \\
\hline Psychrobactera & 0.06 & Caldilineacea & 0.03 & Chrysiogenes & 0.035 \\
\hline Roseovariusa & 0.058 & Flexithrix & 0.03 & Chromatiuma & 0.031 \\
\hline Shinellaa & 0.058 & Alkalilimnicola & 0.03 & Branhamella & 0.031 \\
\hline Fluoribacter & 0.058 & Chromatiuma & 0.03 & Alkalilimnicola & 0.031 \\
\hline Ralstoniaa & 0.058 & Colwelliaa & 0.03 & Flexithrix & 0.031 \\
\hline Carnobacteriaceae_2a & 0.058 & Anaerococcus & 0.03 & Alishewane & 0.031 \\
\hline Curvibactera & 0.058 & Hoefleaa & 0.03 & Burkholderia & 0.031 \\
\hline
\end{tabular}


TABLE 2.

continued

\begin{tabular}{|c|c|c|c|c|c|}
\hline \multicolumn{2}{|c|}{ Diseased Versus Healthy } & \multicolumn{2}{|c|}{ Diseased Versus Healthy on Diseased } & \multicolumn{2}{|c|}{ Healthy Versus Healthy on Diseased } \\
\hline Taxon & $\overline{\text { Coefficient }}$ & Taxon & $\overline{\text { Coefficient }}$ & Taxon & Coefficient \\
\hline Schlegelellaa & 0.058 & Delftia & 0.025 & Turicibacter & 0.031 \\
\hline Diaphorobactera & 0.058 & Hyphomicrobium & 0.025 & Carnobacteriaceae_1 & 0.019 \\
\hline Pseudomonasa & 0.058 & Ahrensia & 0.023 & Ahrensia & 0.013 \\
\hline Asticcacaulisa & 0.058 & Lactococcus & 0.021 & Acidimicrobineae & 0.011 \\
\hline Stenotrophomonasa & 0.058 & Lacinutrix & 0.019 & Delftia & 0.007 \\
\hline Staphylococcusa & 0.058 & Crenothrix & 0.015 & Algibacter & 0.004 \\
\hline Colwellia & 0.058 & Dokdonia & 0.001 & Aquimarina & -0.002 \\
\hline Methylobacteriuma & 0.058 & Achromobacter & -0.274 & Abiotrophia & -0.004 \\
\hline Hyphomicrobium & 0.058 & Cellulophaga & -0.191 & Dokdonia & -0.016 \\
\hline Achromatium & 0.058 & Chrysiogenes & -0.131 & Achromobacter & -0.018 \\
\hline Geopsychrobacter & 0.058 & Abiotrophia & -0.116 & Cellulophaga & -0.046 \\
\hline Brumimicrobium & 0.058 & Fluoribacter & -0.115 & Hyphomicrobium & -0.046 \\
\hline Spirochaetaa & 0.058 & Erythrobacter & -0.109 & Lacinutrix & -0.046 \\
\hline Caldilineacea & 0.058 & Algibacter & -0.091 & Erythromicrobium & -0.063 \\
\hline Agrobacterium & 0.058 & Hydrogenovibrioa & -0.079 & Fluoribacter & -0.066 \\
\hline Cellulophaga & 0.058 & Carnobacteriaceae_1 & -0.068 & Gaetbulibacter & -0.069 \\
\hline Anaerococcus & 0.058 & Acidimicrobineae ${ }^{-}$ & -0.052 & Geothermobacter & -0.072 \\
\hline Roseobactera & 0.049 & Erythromicrobium & -0.052 & Oceanibulbus & -0.09 \\
\hline Nereidaa & -0.263 & Geothermobacter & -0.046 & Kordiimonas & -0.103 \\
\hline Glycomycineae & -0.236 & Oceanibulbus & -0.042 & Devosia & -0.118 \\
\hline Silicibactera & -0.206 & Kordiimonas & -0.037 & Pasteuriaceae_Incertae_Sedis & -0.169 \\
\hline Stappiaa & -0.179 & Gaetbulibacter & -0.015 & Kangiella & -0.258 \\
\hline Cycloclasticus & -0.177 & Devosia & -0.013 & & \\
\hline Sulfitobactera & -0.175 & Pasteuriaceae_Incertae_Sedis & -0.013 & & \\
\hline Phaeobactera & -0.173 & Kangiella - & -0.004 & & \\
\hline Cloacibacteriuma & -0.172 & & & & \\
\hline Erythromicrobium & -0.165 & & & & \\
\hline Salinibactera & -0.153 & & & & \\
\hline Carnobacteriaceae_1 & -0.15 & & & & \\
\hline Branhamella & -0.143 & & & & \\
\hline Turicibacter & -0.141 & & & & \\
\hline Leadbetterellaa & -0.132 & & & & \\
\hline Geothermobacter & -0.124 & & & & \\
\hline Alishewane & -0.12 & & & & \\
\hline Hyphomonasa & -0.115 & & & & \\
\hline Nannocystaceaea & -0.113 & & & & \\
\hline Comamonasa & -0.11 & & & & \\
\hline Algibacter & -0.11 & & & & \\
\hline Piscirickettsiaa & -0.11 & & & & \\
\hline Fluviicola & -0.101 & & & & \\
\hline Propionibacterineaea & -0.099 & & & & \\
\hline Chrysiogenes & -0.099 & & & & \\
\hline Gp4a & -0.096 & & & & \\
\hline Crenothrix & -0.095 & & & & \\
\hline Leucothrix & -0.095 & & & & \\
\hline Methylocapsaa & -0.094 & & & & \\
\hline Krokinobactera & -0.092 & & & & \\
\hline Frateuria & -0.09 & & & & \\
\hline Zobelliaa & -0.088 & & & & \\
\hline Sphingomonasa & -0.088 & & & & \\
\hline Rubellimicrobiuma & -0.086 & & & & \\
\hline Kaistia & -0.083 & & & & \\
\hline Maribactera & -0.081 & & & & \\
\hline Gaetbulibacter & -0.078 & & & & \\
\hline Hoeflea & -0.068 & & & & \\
\hline Hydrogenovibrio & -0.068 & & & & \\
\hline Acidimicrobineae & -0.068 & & & & \\
\hline Saccharophagusa & -0.068 & & & & \\
\hline
\end{tabular}


TABLE 2.

continued

\begin{tabular}{|c|c|c|c|c|c|}
\hline \multicolumn{2}{|c|}{ Diseased Versus Healthy } & \multicolumn{2}{|c|}{ Diseased Versus Healthy on Diseased } & \multicolumn{2}{|c|}{ Healthy Versus Healthy on Diseased } \\
\hline Taxon & Coefficient & Taxon & Coefficient & Taxon & Coefficient \\
\hline Meganemaa & -0.068 & & & & \\
\hline Aminomonas & -0.068 & & & & \\
\hline Nitrospiraa & -0.068 & & & & \\
\hline Thiorhodospiraa & -0.068 & & & & \\
\hline Thioclavaa & -0.068 & & & & \\
\hline Rhodomicrobiuma & -0.068 & & & & \\
\hline Sphingopyxisa & -0.068 & & & & \\
\hline Pibocellaa & -0.068 & & & & \\
\hline Winogradskyellaa & -0.068 & & & & \\
\hline Rhodobacaa & -0.063 & & & & \\
\hline Nitrosospiraa & -0.063 & & & & \\
\hline Microvirgaa & -0.06 & & & & \\
\hline Erythrobacter & -0.06 & & & & \\
\hline Corynebacterineae & -0.059 & & & & \\
\hline Frankineae & -0.051 & & & & \\
\hline Micrococcineaea & -0.05 & & & & \\
\hline
\end{tabular}

A large absolute value of a structure coefficient indicates a close relationship between the coefficient and the function. The sign indicates whether it is correlated positively or negatively to the first disease state (i.e., diseased versus healthy).

more than 100 bacterial members on the cuticle of lobsters from the affected region. However, A. 'homaria' is chitinolytic and could possibly be one of the first colonizers of lesions that are induced by environmental factors in the wild. In addition, Quinn et al. (2012) investigated a prelesion state whereas we are investigating an advanced state of disease with full-blown lesions. The observation that Jannaschia spp. and Aquimarina spp. were correlated with the disease state, albeit very weakly, suggests that these 2 species could be involved in a consortium of first colonizers of carapace lesions. However, other genera, such as Hirschia, Oceanicolaa, and so forth should be considered as possible contributors to the disease (see Table 3).

The role of Aquimarina spp. in the disease lesions is unclear. In the DA that compared the bacteria on cuticle from diseased animals with healthy cuticle from diseased animals, Aquimarina spp. had a smaller structure coefficient (0.156) than that on diseased versus apparently healthy animals. One possible interpretation is that if $A$. 'homaria' was present at the initiation of the disease, then it should be more strongly negatively correlated with the diseased cuticle. Instead, it correlates weakly, and positively, indicating that it discriminates the diseased state from the regions of the carapace that are not affected by the disease directly. Moreover, in the analysis of flora from healthy lobsters versus healthy cuticle on diseased lobsters, the structure coefficient of Aquimarina spp. was negative, and was the lowest value of all the structure coefficients in the structure coefficient table. This indicates a weakly negative correlation; one would expect to find slightly more of this bacterial genus on lesion-free areas of infected lobsters than would be found on lobsters that present no signs of the disease. In addition, the phylogenetic tree indicates that there may be at least 3 species/strains in the genus Aquimarina present in our lobster samples, and the major clades do not cluster with known species and are not correlated directly with the disease state. An alternative hypothesis is that $A$. 'homaria' could initiate the disease lesion, but may be excluded from the mature lesion as other taxa colonize the area.

The Unifrac neighbor joining tree constructed of the microbiome data from each lobster in this study demonstrates that although there is some visible clustering of these samples, there is no clear separation of the clades based on disease class. This is also reflected in the PCO plot, in which we see some clustering of

TABLE 3.

Classification results table for the bacterial flora on the cuticle of diseased lobsters compared with healthy cuticle on diseased lobsters.

\begin{tabular}{lllrrr}
\hline \hline & & \multicolumn{2}{c}{ Predicted Group Membership } \\
\cline { 3 - 6 } & & \multicolumn{1}{c}{ Disease Class } & Diseased & Healthy on Diseased & Total \\
\hline Original & Count & Diseased & 54 & 1 & 55 \\
& & Healthy on diseased & 0 & 1.8 & 32 \\
\cline { 2 - 6 } & $\%$ & Diseased & 98.2 & 100.0 & 100.0 \\
\hline
\end{tabular}

Actual membership is predicted by the discriminant function. The analysis discriminated $98.9 \%$ of original grouped cases correctly. 
TABLE 4.

Classification results table for the bacterial flora on the cuticle of apparently healthy lobsters compared with that on healthy regions of diseased lobsters.

\begin{tabular}{lllrrr}
\hline \hline & & \multicolumn{2}{c}{ Predicted Group Membership } \\
\cline { 3 - 5 } & & \multicolumn{1}{c}{ Disease Class } & Healthy & Healthy on Diseased & Total \\
\hline Original & Count & Healthy & 47 & 0 & 47 \\
& & Healthy on diseased & 0 & 32 & 32 \\
\cline { 2 - 6 } & & Healthy & 100.0 & 0.0 & 100.0 \\
& & Healthy on diseased & 0.0 & 100.0 & 100.0 \\
\hline
\end{tabular}

Actual group membership is compared with that predicted by the discriminant function derived from the analysis. The analysis correctly classified $100 \%$ of the group membership.

the samples based on disease class, but these clusters overlap significantly. This observation could be a result of the fact that all the lobsters were taken in a relatively small geographical region of Narragansett Bay, and presumably exposed to similar environmental factors. The DA indicated that there are 58 genera that are potentially correlated with the disease class, and no single taxa makes a substantial contribution to the metric. Furthermore, most of these taxa are present to some degree in all the sample classes.

In summary, we observed that, of 170 taxa of bacteria, 58 were different in the diseased state, yet none were found to correlate significantly with disease state. Thus, there is no clear correlation of any one taxa with the lesions, indicating that the disease may involve a dysbiotic shift in the shell microbial community resulting from some environmental factor that stresses the lobsters, thereby allowing opportunistic invasion of the carapace by bacteria that normally exist in the carapace biofilm. Although the disease manifests as a bacterial erosion of the cuticle, there is also evidence that the cuticle can be compromised structurally by alkyl phenols that interferes with cross-linking of tyrosine moieties in the premolt cuticle (Laufer et al. 2012). Kunkel et al. (2012) found that lobsters with

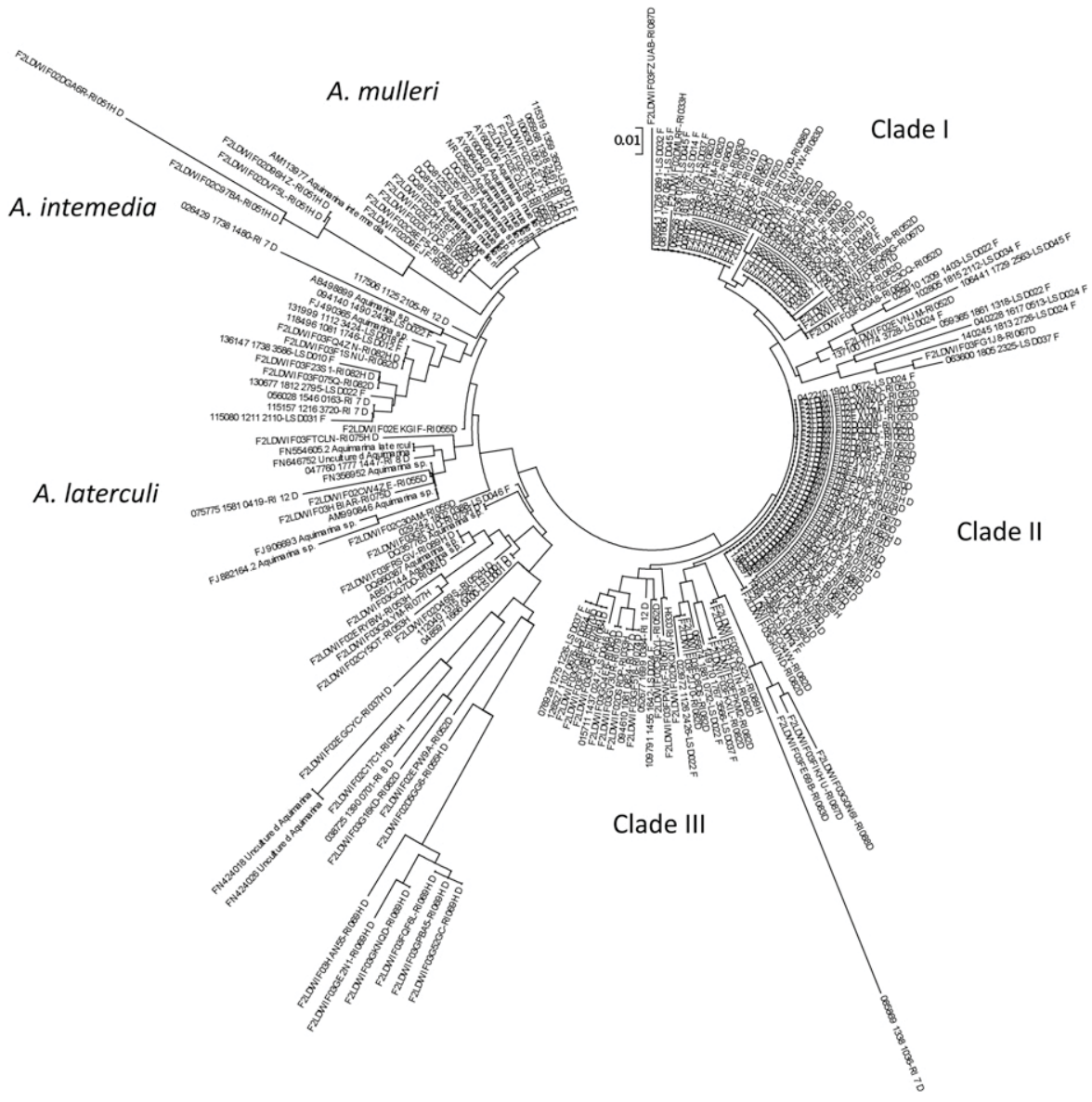

Figure 5. Neighbor joining tree of Aquimarina spp. identified in the carapace microbiome. 
ESD showed signs of erosion or malformation of calcite and apatite structures within the exoskeleton. Both the erosion of calcite and interference of tyrosine cross-linking by alkyl phenols may play a role in making the lobsters more susceptible to ESD. Other factors, such as pollution, increased bottom temperatures, and genetic variation in the lobsters themselves, may also contribute to morbidity.

\section{ACKNOWLEDGMENTS}

This work was supported by the National Marine Fisheries Service as the New England Lobster Research Initiative:
Lobster Shell Disease under NOAA grant NA06NMF4720100 to the University of Rhode Island Fisheries Center. We thank Dr. Kathy Castro and Barbara Somers of the University of Rhode Island for their dedication and expertise in leading the Lobster Shell Disease Initiative, and thank Dr. J. Moss and K. Wheeler, who did the lobster dissections. The views expressed herein are those of the authors and do not necessarily reflect the views of NOAA or any of its subagencies. The U.S. government is authorized to produce and distribute reprints for government purposes, notwithstanding any copyright notation that may appear hereon.

\section{LITERATURE CITED}

Caporaso, J. G., J. Kuczynski, J. Stombaugh, K. Bittinger, F. D. Bushman, E. K. Costello, N. Fierer, A. G. Pena, J. K. Goodrich, J. I. Gordon, G. A. Huttley, S. T. Kelley, D. Knights, J. E. Koenig, R. E. Ley, C. A. Lozupone, D. McDonald, B. D. Muegge, M. Pirrung, J. Reeder, J. R. Sevinsky, P. J. Turnbaugh, W. A. Walters, J. Widmann, T. Yatsunenko, J. Zaneveld \& R. Knight. 2010. QIIME allows analysis of highthroughput community sequencing data. Nat. Methods 7:335-336.

Chistoserdov, A. Y., R. Smolowitz, F. Mirasol \& A. Hsu. 2005. Culturedependent characterization of the microbial community associated with epizootic shell disease lesions in American lobster, Homarus americanus. J. Shellfish Res. 24:741-747.

Cole, J. R., Q. Wang, E. Cardenas, J. Fish, B. Chai, R. J. Farris, A. S. Kulam-Syed-Mohideen, D. M. McGarrell, T. Marsh, G. M. Garrity \& J. M. Tiedje. 2009. The Ribosomal Database Project: improved alignments and new tools for rRNA analysis. Nucl. Acids Res. $37: 141-145$.

Garson, G. D. 2008. Discriminant function analysis. http://faculty. chass.ncsu.edu/garson/PA765/discrim.htm. Accessed May 5, 2010.

Gillevet, P. M. 2006. Multitag sequencing and ecogenomic analysis. EPO 07871488.8; PCT/US2007/084840. Fairfax, VA: BioSpherex LLC.

Klecka, W. R. 1980. Discriminant analysis: quantitative applications in the social sciences. Thousand Oaks, CA: Sage Publications. 72 pp.

Kunkel, J. G., W. Nagel \& M. J. Jercinovic. 2012. Mineral fine structure of the American lobster cuticle. J. Shellfish Res. 31:515-526.
Laufer, H., M. Chen, M. Johnson, N. Demir \& J. M. Bobbitt. 2012. The effect of alkyl phenols on lobster shell hardening. J. Shellfish Res. 31:555-562.

Lozupone, C. \& R. Knight. 2005. UniFrac: a new phylogenetic method for comparing microbial communities. Appl. Environ. Microbiol. 71:8228-8235.

Quinn, R. A., A. Metzler, R. M. Smolowitz, M. Tlusty \& A. Y. Chistoserdov. 2012. Exposures of Homarus americanus shell to three bacteria isolated from naturally occurring epizootic shell disease lesions. J. Shellfish Res. 31:485-493.

Shields, J. D., K. N. Wheeler \& J. A. Moss. 2012. "Histological assessment of the lobsters (Homarus americanus) in the "100 lobsters" project." J. Shellfish Res. 31:439-447.

Smolowitz, R., A. Y. Chistoserdov \& A. Hsu. 2005. A description of the pathology of epizootic shell disease in the American lobster, Homarus americanus, H. Milne Edwards 1837. J. Shellfish Res. 24 749-756.

Suzuki, M., M. S. Rappe \& S. J. Giovannoni. 1998. Kinetic bias in estimates of coastal picoplankton community structure obtained by measurements of small-subunit rRNA gene PCR amplicon length heterogeneity. Appl. Environ. Microbiol. 64:4522-4529.

Tlusty, M. F. \& A. Metzler. 2012. Relationship between temperature and shell disease in laboratory populations of juvenile American lobsters (Homarus americanus). J. Shellfish Res. 31:533-541. 